\title{
A SHORT PROOF OF A THEOREM OF ADJAN
}

\author{
LOUXIN ZHANG
}

(Communicated by Warren J. Wong)

\begin{abstract}
In this note, using the technique of rewriting, we give a short proof of a theorem of Adjan: the word problem is decidable for special one-relator monoids $(A ; w=e)$.
\end{abstract}

The word problem for one-relator monoids is still open, in spite of the fact that the word problem for one-relator groups has been solved positively by Magnus [2]. A general result on the word problem for one-relator monoids is the following one due to Adjan [1]:

Theorem 1. The word problem is decidable for special one-relator monoids $(A ; w=e)$.

Let $A$ be a finite set, and let $A^{*}$ be the free monoid generated by $A$, the identity of which is denoted by $e$. If $x, y \in A^{*}$, by $x=y$ we mean that $x$ and $y$ are the same element. Let $R$ be a relation of $A^{*}$. The reduction $\rightarrow_{R}^{*}$ induced by $R$ is the reflexive, transitive closure of the relation $\rightarrow_{R}$ defined by $u \rightarrow_{R} v$ iff $\exists x, y \in A^{*}, \quad(l, r) \in R$ such that $u=x l y, v=x r y$. By $\leftrightarrow_{R}^{*}$ we denote the symmetric, transitive closure of $\rightarrow_{R}^{*}$, which is the smallest congruence containing $R$. Let $w \in A^{*}$, the special one-relator monoid $M=$ $(A ; w=e)$ is the quotient of $A^{*}$ by $\leftrightarrow_{R}^{*}$, where $R=\{(w, e)\}$.

A relation $R$ on $A^{*}$ is called Noetherian if there exists no infinite sequence of reductions of the form $u_{1} \rightarrow_{R} u_{2} \rightarrow_{R} \cdots$; it is called confluent if for any $x, y \in A^{*}$ such that $x \leftrightarrow_{R}^{*} y, x \rightarrow_{R}^{*} z$ and $y \rightarrow_{R}^{*} z$ for some $z \in A^{*}$.

If a relation $R$ on $A^{*}$ is Noetherian and confluent, then each congruence class $[w]_{R}=\left\{u \in A^{*} \mid u \leftrightarrow_{R}^{*} w\right\}$ of $w \bmod R$ contains exactly one element $\bar{w}$ such that there exists no element $v$ satisfying $\bar{w} \rightarrow_{R} v$. Define $\bar{w}$ to be the norm form of $w$. Thus, if $R$ is Noetherian and confluent and there is an algorithm to find the norm form for each element in $A^{*}$, then the word problem is decidable for the monoid $A^{*} / \leftrightarrow_{R}^{*}$ since $x=y$ in $A^{*} / \leftrightarrow_{R}^{*}$ iff $\bar{x}$ and $\bar{y}$ are identical.

Given a special one-relator monoid $M=(A ; w=e)$, we construct a sequence of sets $C_{i}$ as follows:

$$
\begin{aligned}
& C_{1}=\{w\}, \\
& C_{i+1}=C_{i} \cup\left\{x y \mid x \in W\left(C_{i}\right) \& y x \in C_{i}\right\} \cup\left\{z x \mid x \in W\left(C_{i}\right) \& x z \in C_{i}\right\},
\end{aligned}
$$

for $i \geq 1$, where $W\left(C_{i}\right)$ denotes the set of all elements that are both left and

Received by the editors November 20, 1990 and, in revised form, February 11, 1991 .

1980 Mathematics Subject Classification (1985 Revision). Primary 20M05, 03D03, 03D40. 
right factors of elements of $C_{i}$. Obviously, $C_{1} \subseteq C_{2} \subseteq \cdots \subseteq C_{i} \subseteq C_{i+1} \subseteq \cdots$. On the other hand, for all elements $u \in C_{j}, \quad u$ has the same length as $w$. Thus, there exists $k$ such that $C_{k}=C_{k+j}$ for $j \geq 1$. Denote the set of elements in $W\left(C_{k}\right)$ such that no proper right factor of them are in $W\left(C_{k}\right)$ by $E(M)$.

Proposition 1. Let $x, y, z \in A^{*}$ and $M=(A ; w=e)$ be a special one-relator monoid. Then

(1) $x y, y z \in E(M) \Rightarrow y=e$ or $x=z=e$;

(2) $x y, y z \in E(M)^{*} \Rightarrow y \in E(M)^{*}$.

Proof. (1) Let $x y, y z \in E(M)$. Suppose $y \neq e$. Then, since $x y \in E(M)$, there exists $H \in A^{*}$ such that $H x y \in C_{k}$. Symmetrically, there exists $F \in A^{*}$ such that $y z F \in C_{k}$; so, $y \in W\left(C_{k}\right)$. Since $x y \in E(M), y \in W\left(C_{k}\right)$ implies $x y=y$ and so $x=e$. Similarly, $z=e$.

(2) Let $x y, y z \in E(M)^{*}$. Suppose $x y=x_{1} x_{2} \cdots x_{k}$, where $x_{j} \in E(M)$ for each $j$. Then $y=x_{i}^{\prime \prime} x_{i+1} \cdots x_{k}$ for some nonempty right factor $x_{i}^{\prime \prime}$ of $x_{i}$. Since $y z=x_{i}^{\prime \prime} x_{i+1} \cdots x_{k} z \in E(M)^{*}$, there is a $c \in E(M)$ that overlaps with $x_{i}^{\prime \prime}$, say $c=c_{1} c_{2}$ and $x_{i}^{\prime \prime}=v c_{1}$, where $c_{1} \neq e$ and $c_{2}, v \in A^{*}$. Since $x_{i}=x_{i}^{\prime} x_{i}^{\prime \prime}=x_{i}^{\prime} v c_{1}$ and $c=c_{1} c_{2}, \quad c_{1} \neq e$ implies $x_{i}^{\prime} v=e$ by (1) and so $y \in E(M)^{*}$.

Let $E(M)=\left\{x_{1}, x_{2}, \ldots, x_{n}\right\}$. Introduce an alphabet $B$ in bijection with $E(M)$ (say, through $\varphi: E(M) \rightarrow B$ ). Since $w$ is a product of elements in $E(M), \varphi(w)$ is defined. We say that the monoid presentation $(B ; \varphi(w)=e)$ is obtained from the monoid presentation $(A ; w=e)$ by the technique of rewriting. Let $s \in E(M)$ and $w=s t$ for some $t \in A^{*}$, then $t \in E(M)^{*}$ and $t s \leftrightarrow_{\{(w, e)\}}^{*} e$; so $\varphi(t) \varphi(s) \leftrightarrow_{\{(\varphi(w), e)\}}^{*} e$. Thus, the presentation $(B ; \varphi(w)=e)$ presents a one-relator group, say $G$.

Using the set $E(M)$ and the group $G$, we define a relation $R=R(M)$ over $A^{*}$ in the following way:

$$
R=\left\{(u, v) \mid u, v \in E(M)^{*}: u>v \& \varphi(u)=\varphi(v) \text { in } G\right\},
$$

where $<$ is a linear order defined by: $x<y$ iff $|x|<|y|$ or $|x|=|y|$ and $x<_{\text {lex }} y$. Here $<_{\text {lex }}$ denotes the lexicographical order on $A^{*}$ induced by a given linear order on $A$.

Lemma 1. Let $u \in A^{*}$ with $|u|<k=\max _{x \in E(M)}|x|$. Then $u$ is irreducible $\bmod R$, i.e., there is no $v \in A^{*}$ such that $u \rightarrow_{R} v$.

Proof. Let $u \in A^{*}$ with $|u|<k$. Suppose $u$ is not irreducible $\bmod R$. Then, there are $u^{\prime}, u^{\prime \prime} \in A^{*}$ and $x, y \in E(M)^{*}$ such that $u=u^{\prime} x u^{\prime \prime}$ and $(x, y) \in R$. Since $|y| \leq|x|<k$, at least the letter corresponding to the word in $E(M)$ with the maximum length $k$ does not occur in both $\varphi(x)$ and $\varphi(y)$, so by Freiheitssatz for one-relator groups [3], $\varphi(x)=\varphi(y)$ in $G$ implies $\varphi(x)=$ $\varphi(y)$, which in turn implies $x=y$ from Proposition 1(1), a contradiction.

Proposition 2. Let $T=\{(w, e)\}$. Then $R$ is Noetherian, confluent, and equivalent to $T$, i.e., $\leftrightarrow_{R}^{*}=\leftrightarrow_{T}^{*}$.

Proof. Since $<$ is a linear ordering on $A^{*}$, since this ordering is compatible with the product in $A^{*}$ and since $u>v$ for each $(u, v) \in R, R$ must be Noetherian. 
To show $R$ is confluent, we use Theorem 1 in [4]. For condition (1), let $(x y, p),(y z, q)$ be two rules in $R$. Since $x y, y z \in E(M)^{*}$, by Proposition $1, x, y, z \in E(M)^{*}$. Thus $x q, p z \in E(M)^{*}$. On the other hand, $\varphi(x q)=$ $\varphi(x) \varphi(q)={ }_{G} \varphi(x) \varphi(y z)=\varphi(x y) \varphi(z)={ }_{G} \varphi(p) \varphi(z)=\varphi(p z)$. Since $<$ is a linear ordering, $x q=p z, x q<p z$, or $p z<x q$. Then, by the definition of $R$, either $(x q, p z)$ or $(p z, x q)$ must be a rule in $R$, or else $x q=p z$. For condition (2), if $(x y z, p)$ and $(y, q)$ are two rules in $R$, since $x y z \in E(M)^{*}$ and $y, q \in E(M)^{*}$, by Proposition 1, we have either (1) $x, y, z \in E(M)^{*}$ or (2) $x=c_{1} c_{2} \cdots c_{s} F$ and $z=H d_{1} d_{2} \cdots d_{t}$ and $F y H \in E(M)$ for some $F, H \in A^{+}=A^{*}-\{e\}$.

Case (1). We have $x q z \in E(M)^{*}, \varphi(x q z)=\varphi(x) \varphi(q) \varphi(z)$ ${ }_{G} \varphi(x) \varphi(y) \varphi(z)={ }_{G} \varphi(p)$. So either $(x q z, p)$ or $(p, x q z)$ must be rule in $R$ or $x q z=p$.

Case (2). Since $F, H \in A^{+},|y|<k=\max _{x \in E(M)}|x|$, by Lemma 1 , which implies $y$ is irreducible $\bmod R$, a contradiction.

Therefore, $R$ is confluent.

Since $w \in E(M)^{*}$ and $\varphi(w)=e$ in $G$, we have $(w, e) \in R$, i.e., $T \subseteq R$. On the other hand, for each rule $(u, v) \in R, \varphi(u)=\varphi(v)$ in $G$ implies $u \leftrightarrow_{T}^{*} v$, so $\leftrightarrow_{R}^{*} \subseteq \leftrightarrow_{T}^{*}$. Hence $R$ is equivalent to $T$.

Proof of Theorem 1. Let $M$ be a special one-relator monoid $(A ; w=e)$. Since $R$ is Noetherian, confluent and equivalent to $T=\{(w, e)\}$, given two elements $u, v \in A^{*}$, in order to decide whether $u=v$ in $M$, i.e., $u \leftrightarrow_{T}^{*} v$, we need only to find the norm forms $\bar{u}$ and $\bar{v} \bmod R$ of $u$ and $v$ respectively, and then compare $\bar{u}$ to $\bar{v}$. If $\bar{u}$ and $\bar{v}$ are identical, then $u \leftrightarrow_{T}^{*} v$; otherwise, $u \leftrightarrow \leftrightarrow_{T}^{*} v$.

Since $G$ is a one-relator group, it has a decidable word problem. So, given two elements $x, y \in E(M)^{*}$, such that $x>y$, whether or not $\varphi(x)={ }_{G} \varphi(y)$ can be decided. Note that there are only finitely many words in $E(T)^{*}$ less than $x$ w.r.t. $<$. Thus we can find $\bar{u}$ and $\bar{v}$ in a finite number of steps. Therefore the word problem is decidable for $M$.

\section{ACKNOWLEDGMENT}

The author thanks the referee for a detailed report, which was very helpful in preparing the final version of this note.

\section{REFERENCES}

1. S. Adjan, Defining relations and algorithmic problems for groups and semigroups, Trudy Mat. Inst. Steklov 85 (1966); English. transl. in Proc. Steklov. Inst. Math. 85 (1966).

2. W. Magnus, Das identity problem fur grouppen mit einer definierenden relation, Math. Ann. 106 (1932), 295-307.

3. W. Magnus, A. Karrass, and D. Solitar, Combinatorial group theory, Wiley, New York, 1966.

4. R. McNaughton and P. Narendran, Special monoids and special Thue systems, J. Algebra 108 (1987), 248-255.

Department of Pure Mathmatics, University of Waterloo, Waterloo, Ontario, CanAda, N2L 3G1

Department of Computer Science, University of Waterloo, Waterloo, Ontario, Canada, N2L 3G1 